Revista Brasileira de Engenharia Biomédica, v. 25, n. 2, p. 101-105, agosto 2009

(C) SBEB - Sociedade Brasileira de Engenharia Biomédica ISSN 1517-3151

http://dx.doi.org/10.4322/rbeb.2012.070

Comunicação Técnica

Recebido em 14/11/2007, aceito em 01/09/2009

\section{Avaliação de desempenho em um medidor eletromagnético de baixo custo desenvolvido para aplicações em simulação cardiovascular}

Performance evaluation on low
cost electromagnetic flow meter for
cardiovascular simulation system
application

Robson Colantonio* Julio César Lucchi

Instituto Mauá de Tecnologia, Departamento de Pós-graduação, Praça Mauá, 01 09580-900 São Caetano do Sul, SP - Brasil E-mail: monumentalengenharia@yahoo.com.br

\section{Aron José Pazin de Andrade}

Instituto Dante Pazzanese de Cardiologia, Departamento de Bioengenharia.

*Autor para correspondência
Resumo

Técnicas cirúrgicas, próteses e dispositivos implantáveis utilizados pela cardiologia estão em constante desenvolvimento. Alguns institutos de pesquisas e universidades desenvolvem simuladores hidrodinâmicos com capacidade de reproduzir os níveis de pressão e vazão do sistema cardiovascular humano, com pistões, válvulas, câmaras de complacência, dispositivos para ajustes e medição de pressão e vazão. Este artigo apresenta o desenvolvimento e a avaliação de um fluxômetro eletromagnético (FE). O custo elevado de um fluxômetro por ultrassom (FUS) pode inviabilizar algumas pesquisas com simuladores; desta forma, uma alternativa com menor custo é apresentada neste trabalho. Baseado na indução eletromagnética para medição de vazão em um fluido iônico, o FE desenvolvido mostrou ser uma solução de baixo custo. $\mathrm{O}$ transdutor foi montado em um tubo de plástico com diâmetro interno de 9,5 mm, excitado por um campo magnético de 0,35 T, possui 4 eletrodos que, ligados a um circuito eletrônico, fornecem tensão elétrica proporcional à velocidade do fluido. A equação da tensão induzida, técnicas para calibração, ensaios e resultados são apresentados. Para a realização dos ensaios, foram utilizados um simulador cardiovascular e um FUS como referência. Os dados foram obtidos por hardware e software da National Instruments. Dentre os resultados, destaca-se o baixo desvio padrão de 0,043 L/ min do FE para uma vazão de 3,56 L/min. O maior erro porcentual relativo foi de 1,49 $\pm 1,25 \%$. Em um dos gráficos de vazão do $\mathrm{FE}$ observou-se um comportamento não monotônico, confirmado por uma análise da resposta em freqüência, observando-se maior amplitude na segunda, terceira e quarta harmônicas. Os resultados indicaram que o FE pode substituir o FUS em aplicações com simuladores hidrodinâmicos.

Palavras-chave: Medida de vazão, Fluxômetro eletromagnético, Simulador cardiovascular.

\section{Abstract}

Implantable devices, prosthesis and their associated surgical techniques in cardiology are constantly under development. Many research groups have been using hydrodynamic simulators as a tool to assist the development in cardiovascular area, capable to mimic pressure and flow found in human cardiovascular system. They are made with plastic tubes, compliances chambers, valves, moving diaphragms and clamps for pressure and flow adjustments. The high cost of ultrasonic flow meters (UF) may difficult, for some research groups, the development of their own mock systems. This paper presents a simple and low cost electromagnetic flow meter (EF) useful for those cardiovascular simulation systems. EF measures flow of ionic fluids based on electromagnetic induction. A special transducer was assembled directly on a plastic tube with $9.5 \mathrm{~mm}$ of inner diameter. The transducer has 4 electrodes and, when excited by a magnetic field of 0.35 tesla, detects electric tension that is proportional to the fluid velocity. In this paper, induced electric potential equation, calibration techniques, performance tests and results are presented. A cardiovascular simulation system was used as test setup with an UF (Transonic Systems Inc.) as comparative flow meter. The signals were acquired by a PCI-6036E card and processed with LabView $^{\circledast} 7.1$ (National Instruments). The results from EF showed a standard deviation (SD) of $0.043 \mathrm{~L} / \mathrm{min}$ for a flow of $3.56 \mathrm{~L} / \mathrm{min}$. The largest relative error was of $1.49 \pm 1.25 \%$. In one flow signal from the $E F$, the non monotonic behavior suggests a rich harmonic signal, which was confirmed by an analysis in the frequency domain. In vitro performance tests indicated that our EF can substitute the commercial available UF for hydrodynamic simulator application.

Keywords: Blood flow, Electromagnetic flow meter, Cardiovascular simulation system. 


\section{Introdução}

A cardiologia, em especial a cirurgia cardiovascular, é uma área que apresentou nas últimas décadas um grande desenvolvimento no Brasil. Técnicas cirúrgicas e dispositivos para implantes como válvulas e próteses vasculares estão constantemente em aperfeiçoamento e testes (Andrade et al., 1999). Para realização de ensaios, estes dispositivos podem ser submetidos a simuladores hidrodinâmicos com capacidade de reproduzir os níveis de pressão e vazão observados em vários pontos do sistema cardiovascular humano como, por exemplo, a simulação dos grandes vasos, a veia pulmonar e artéria aorta (Felipini et al., 2005; Lonardoni, 2006; Timms et al., 2005).

Muitos institutos de pesquisas e universidades desenvolvem simuladores, geralmente compostos por pistões, válvulas, manômetros, termostato, câmaras de complacência e instrumentos para medição de pressão e vazão.

A medição de vazão em tempo real é muito importante nestes simuladores, e fluxômetros por ultrassom (FUS), desenvolvidos para serem utilizados em cirurgias, podem ser usados apresentando boa eficiência. Em alguns casos, o elevado custo dos FUS pode inviabilizar a aplicação, justificando a importância de pesquisas para alternativas com menor custo. Existem medidores eletromagnéticos de vazão ou fluxômetros eletromagnéticos (FE) que são muito utilizados na área médica em bombas centrífugas para circulação extracorpórea (Carr e Brown, 1988).

Na medição de vazão em fluidos iônicos com FE, fenômeno descoberto por Michael Faraday e Joseph Henry, observa-se que a variação do fluxo magnético ocorre de modo similar ao movimento de um condutor retilíneo dentro de um campo magnético fixo, ou seja, o deslocamento do fluido dentro do vaso que o conduz. Um campo magnético aplicado no sentido perpendicular ao vaso por onde circula o fluido, induz neste uma diferença de potencial elétrico que pode ser medida por dois eletrodos. Um circuito eletrônico amplifica esta diferença obtendo resultado proporcional à vazão dentro do transdutor.

Esta pesquisa apresenta uma solução de baixo custo para a medida de vazão em tempo real, a construção e os ensaios de um fluxômetro e um transdutor de fluxo baseado na metodologia eletromagnética (Bevir, 1970; Hemp e Wyatt, 1981). Os ensaios foram realizados em um simulador cardiovascular e os resultados obtidos foram comparados com um FUS destinado ao uso médico (Carr e Brown, 1988; Dantas e Costa, 2000).

\section{Materiais e Métodos}

O protótipo do transdutor, apresentado na Figura 1, foi montado em um tubo de plástico PVC cristal (Plastic Mangueiras) código CE20C5, com diâmetro interno de 9,5 $\mathrm{mm}$, parede de $2 \mathrm{~mm}$, comprimento de $70 \mathrm{~mm}$ e capacidade máxima de pressão equivalente a $3.600 \mathrm{mmHg}$. Foram inseridos, sob pressão, 4 eletrodos de latão com $1 \mathrm{~mm}$ de diâmetro, $5 \mathrm{~mm}$ de comprimento revestidos com uma película em ouro de $10 \mu \mathrm{m}$ de espessura, onde foram soldados cabos do tipo coaxial paralelo para tomada dos sinais de medição e retorno dos sinais de referência. $\mathrm{O}$ campo magnético aplicado foi de 0,35 T, verificado com um medidor (Instrutherm, modelo DRE-025). Este campo foi obtido com 2 imãs permanentes constituídos por uma liga de neodímio, ferro e boro $\left(\mathrm{Nd}_{2} \mathrm{Fe}_{14} \mathrm{~B}\right)$, com as medidas de $15 \mathrm{~mm}$ de largura, $25 \mathrm{~mm}$ de comprimento e $5 \mathrm{~mm}$ de espessura e fixados paralelos a uma distância máxima de $18 \mathrm{~mm}$ entre eles, de forma a fechar o circuito magnético. Os custos do transdutor eletromagnético e do amplificador foram avaliados em US\$200,00.

O circuito eletrônico apresentado na Figura 2 foi projetado para ter as características com alto ganho na entrada diferencial, rejeição do sinal em modo comum e ajustes de offset para compensar o nível CC observado entre os eletrodos de medição, na ordem de $1 \mathrm{mV}$, para vazão nula. $\mathrm{O}$ ruído em $60 \mathrm{~Hz}$ foi minimizado com o uso de realimentação do sinal de modo comum, retornando-se este nos 2 eletrodos de referência do transdutor eletromagnético, usando uma topologia similar àquela utilizada em amplificadores de eletrocardiografia (Carr e Brown, 1988).

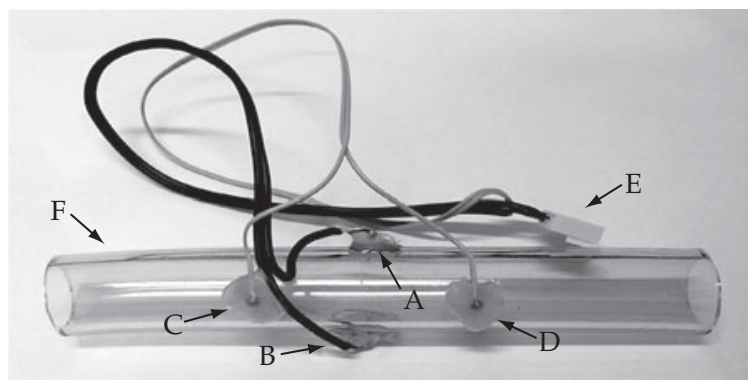

Figura 1. Transdutor eletromagnético. (A) e (B) Eletrodos de medição, (C) e (D) Eletrodos de referência, (E) Conector, (F) Tubo em plástico PVC. Figure 1. Electromagnetic transducer. (A) and (B) Electrodes for measurement, (C) and (D) Reference electrodes, (E) Connector, (F) Plastic tube (PVC). 


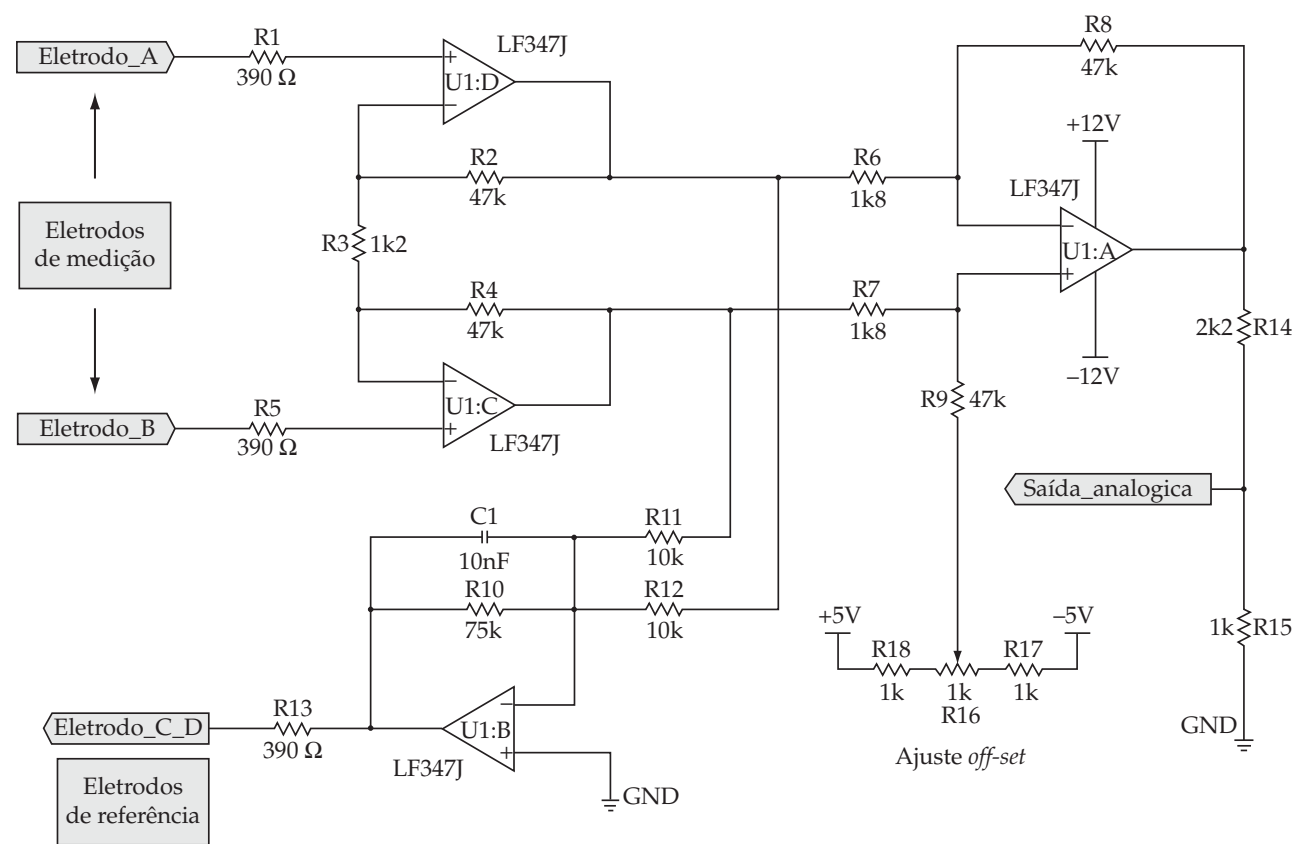

Figura 2. Amplificador utilizado no fluxômetro eletromagnético. Figure 2. Amplifier used for electromagnetic flow meter.

O ganho total do amplificador, na ordem de 647, é dado pela equação 1 (Cathey, 1994):

$G=\left(1+\frac{2 R_{2}}{R_{3}}\right) \cdot\left(\frac{R_{8} \cdot R_{15}}{R_{6}\left(R_{14}+R_{15}\right)}\right)$

$\mathrm{O}$ transdutor eletromagnético apresenta boa linearidade, e a diferença de potencial $E(t)$ presente entre os eletrodos de medição é dada (em volts) pela equação 2 (Delmee, 2003):

$E(t)=\frac{4 B \cdot d_{e} \cdot Q}{\pi \cdot d^{2}}$

onde $B$ é a densidade de fluxo magnético, $d_{e}$ é a distância entre os eletrodos, $Q$ a vazão do fluido e $d$ o diâmetro interno do tubo do transdutor. Todas as unidades estão no sistema internacional (SI).

Com o objetivo de avaliar o desempenho do FE, utilizou-se como referência um FUS (Transonic Systems Inc., modelo HT110), que utiliza a técnica de ultrassom e tempo de trânsito (Carr e Brown, 1988; Christensen, 1988). O FUS apresenta faixa de leitura de $0,1 \mathrm{~mL} / \mathrm{min}$ a $200 \mathrm{~L} / \mathrm{min}$, freqüência de trabalho do transdutor na faixa de $300 \mathrm{kHz}$ a $7,2 \mathrm{MHz}$, erro de $\pm 5 \%$ do valor da leitura e filtro de saída em $5 \mathrm{~Hz}$. Este medidor tem custo na faixa de US\$ 8.000,00. Os fluxômetros foram ligados na mesma mangueira, em plástico PVC código CE20C5, posicionados a uma distância de $400 \mathrm{~mm}$. Foi utilizado um simulador cardiovascular composto por um atuador eletromecânico para simulação da câmara cardíaca do ventrículo esquerdo, onde um motor de corrente contínua sem escovas aciona a câmara de bombeamento através do eixo manivela, válvulas, câmara de complacência, mangueiras, resistência elétrica para aquecimento do fluido e termostato para controlar a temperatura.

Os sinais dos eletrodos, proporcionais à vazão, foram coletados por uma placa PCI-6036E (National Instruments,) tratados pelo software LabView $^{\oplus}$ versão 7.1 (National Instruments) com o uso de um computador PC (Pentium 4, 3 GHz, 512 MB RAM). A placa apresenta custos na faixa de US\$1.800,00 e o software US\$1.500,00.

A taxa de amostragem para os sinais coletados foi arbitrariamente ajustada em 100 amostras por segundo, os dados do FE foram submetidos a um filtro digital do tipo média móvel com 9 coeficientes (Smith, 1999) e a freqüência de corte em $5 \mathrm{~Hz}$ (Antoniou, 2000; Oppenheim e Schafer, 1998). Os valores médios de vazão apresentados foram obtidos por meio de integração numérica pelo método dos trapézios, o erro porcentual foi calculado em relação ao FUS.

Para a calibração dos fluxômetros utilizou-se uma bomba de fluxo contínuo ligada na mangueira onde estavam acoplados. Os ajustes do FE e do FUS foram realizados em duas etapas. Inicialmente o sistema foi ajustado para manter a temperatura de $37^{\circ} \mathrm{C}$ e uma vazão de $2 \mathrm{~L} / \mathrm{min}$. Após 30 minutos de funcionamento, a bomba foi desligada (vazão nula) e o ajuste do ponto zero do FE foi realizado no potenciômetro de offset (Hill, 1982). O FUS possui a função de ajuste automático. A bomba foi religada e ajustada para uma vazão de $5 \mathrm{~L} / \mathrm{min}$, medida no FUS. Foi ajustado o valor do ganho do FE criando-se equivalência entre os medidores. 
Tabela 1. Valores da média, desvio padrão e erro porcentual relativo entre o FUS e o FE observados em 30 amostras nos 2 ensaios. Vazão em L/min. Table 1. Mean flow values and standard deviation in L/min, relative error in percentage between EF and UF observed during 2 experiments and 30 samples.

\begin{tabular}{ccccc}
\hline & FE sem filtro & FE com filtro & FUS & Erro Percentual \\
& (Vazão L/min) & (Vazão L/min) & (Vazão L/min) & $(\%)$ \\
\hline Cálculo Utilizado & Média \pm desvio padrão & Média \pm desvio padrão & Média \pm desvio padrão & Média \pm desvio padrão \\
$1^{\circ}$ Ensaio & $2,035 \pm 0,026$ & $2,035 \pm 0,027$ & $2,059 \pm 0,014$ & $1,49 \pm 1,25$ \\
$2^{\circ}$ Ensaio & $3,560 \pm 0,044$ & $3,560 \pm 0,043$ & $3,578 \pm 0,016$ & $1,05 \pm 0,71$ \\
\hline
\end{tabular}

O simulador cardiovascular foi utilizado para gerar os sinais dos ensaios dos fluxômetros. A simulação dos pulsos de pressão e vazão foi obtida ajustando-se a câmara de complacência e a rotação do motor responsável pelo bombeamento (Lonardoni, 2006).

\section{Resultados}

Durante os 60 minutos que duraram os ensaios não foi observada alteração nos valores do ponto zero no FE e nos valores médios de vazão. A Tabela 1 apresenta 2 ensaios com os valores médios e o desvio padrão observado em 30 pulsos gerados pelo simulador.

A Figura 3 apresenta as formas de onda do segundo ensaio com o simulador ajustado para uma vazão média de 3,58 L/min com freqüência de 93,8 BPM. Foram observados picos de vazão na ordem de $10 \mathrm{~L} /$ min e uma vibração mecânica no simulador. Os instantes referentes aos picos máximos de vazão $(0,56 \mathrm{~s}, 0,62 \mathrm{~s}$ e $1,20 \mathrm{~s}$, respectivamente os pontos $A$, $B$ e $C$ ) foram utilizados para o cálculo do atraso entre as medidas e a freqüência dos batimentos. Foi observado que o sinal do FUS está atrasado em $60 \mathrm{~ms}$ em relação ao sinal do FE submetido ao filtro digital. A máxima pressão observada entre os transdutores foi de $200 \mathrm{mmHg}$.

\section{Discussão e Conclusão}

É esperado um resultado linear da diferença de potencial elétrico em relação à vazão, conforme as equações 1 e 2 . A possibilidade do atraso entre os sinais dos fluxômetros ter sido causada pela distância entre os transdutores foi descartada, já que um ensaio invertendo-se o sentido do fluxo apresentou o mesmo resultado. O procedimento de calibração do FE pode ser feito com um rotâmetro ou qualquer método de medição de vazão, evitando-se em uma aplicação de bancada o uso do FUS.

Com o objetivo de observar as formas de onda dos sinais de vazão, os ensaios, resultados e análises comprovam a capacidade do FE em substituir o FUS apresentado.

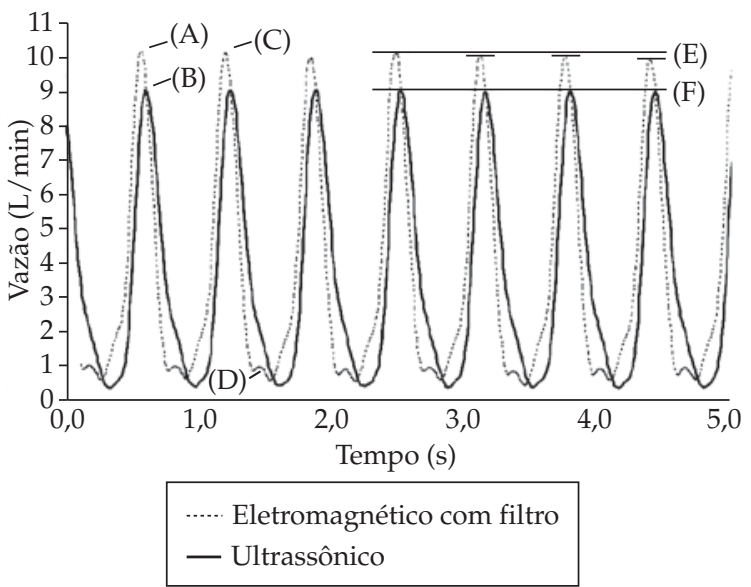

Figura 3. Formas de onda do $2^{\circ}$ ensaio com vazão de 3,58 L/min e 93,8 BPM. (A) Tempo de 0,56 s, (B) Tempo de 0,61 s, (C) Tempo de 1,20 s, (D) Detecção de sinal não monotônico, (E) Variação de amplitude no sinal de Vazão do $F E$, (F) Amplitude estável do sinal de Vazão do FUS.

Figure 3. Flow waveform from second experiment with mean flow of $3.58 \mathrm{~L} / \mathrm{min}$ and 93.8 BPM. (A) Time at $0.56 \mathrm{~s}$, (B) Time at $0.62 \mathrm{~s},(C)$ Time at $1.20 \mathrm{~s}$, (D) Non monotonic behavior of the signal, (E) Amplitude variation on flow wave form from EF, (F) Stable amplitude on flow wave form from UF.

\section{Agradecimentos}

Os autores agradecem ao Instituto Dante Pazzanese pelo apoio cedendo o material necessário para os ensaios, equipamentos de medição e acompanhamento no desenvolvimento da pesquisa.

\section{Referências}

ANDRADE, A. J. P.; OHASHI, Y.; LUCCHI, J. C.; NICOLOSI, D.; DINKHUYSEN, J. J.; BISCEGLI, J.; ARRUDA, A. C. F.; CUNHA, W. C.; NOSÉ Y. Testes in vitro e in vivo com o Coração Artificial Auxiliar (CAA): um novo modelo de coração artificial totalmente implantável e heterotópico. Revista Brasileira de Cirurgia Cardiovascular, v. 14, n. 2, p. 128-134, 1999.

ANTONIOU, A. Digital Filters: Analysis, Design, and Applications. New York: McGraw-Hill, 2000.

BEVIR, M. K. The theory of induced voltage electromagnetic flowmeters. Journal of Fluid Mechanics Digital Archive, v. 43, n. 3, p. 577-590, 1970. 
CARR, J. J.; BROWN, J. M. Introduction to Biomedical Equipment Technology. New Jersey: Prentice-Hall, 1998.

CATHEY, J. J. Dispositivos e circuitos eletrônicos. São Paulo: Makron Books, 1994.

CHRISTENSEN, D. A. Ultrasonic Bioinstrumentation. New York: John Wiley \& Sons, 1988.

DANTAS, R. G.; COSTA, E. T. Ultrasonic pulsed Doppler blood flowmeter for use in extracorporeal circulation. Artificial Organs, v. 24, n. 3, p. 198-201, 2000.

DELMEE, G. J. Manual de Medição de Vazão. São Paulo: Edgard Blucher, 2003.

FELIPINI, C. L.; LUCCHI, J. C.; ANDRADE, A. J. P. Simulador eletro-fluidodinâmico do sistema cardiovascular para pesquisa na área de circulação assistida. In: INTERNATIONAL CONGRESS OF MECHANICAL ENGINEERING - COBEM 2005, 18, nov. 2005, Ouro Preto. Proceedings..., p. 1-7.

HEMP, J.; WYATT, D. G. A basis for comparing the sensitivities of different electromagnetic flowmeters to velocity distribution. Journal of Fluid Mechanics Digital Archive, v. 112, p. 189-201, 1981.

HILL, R. D. A rapid technique for dynamic calibration of electromagnetic flowmeters. Journal of Applied Physiology, v. 53, n. 1, p. 294-296, 1982.

LONARDONI, J. A. C. Desenvolvimento de um Simulador da Mecânica Cardiovascular Humana controlada pelo Mecanismo Reflexo Baroceptor. São Paulo, 2006. 143 p. Tese - (Mestrado em Engenharia Mecatrônica), Escola Politécnica, Universidade de São Paulo - USP.

OPPENHEIM, A. V.; SCHAFER, R. W. Discrete-Time Signal Processing. New Jersey: Prentice Hall, 1998.

SMITH, S. W. The Scientist and Engineer's Guide to Digital Signal Processing. San Diego: California Technical Publishing, 1999.

TIMMS, D.; HAYNE, M.; MCNEIL, K.; GALBRAITH, A. A complete mock circulation loop for the evaluation of left, right, and biventricular assist devices. Artificial Organs, v. 29, n. 7, p. 564-572, 2005. 
